\title{
Recombinant expression in E. coli of human FGFR2 with its transmembrane and extracellular domains
}

\author{
Adam Bajinting ${ }^{1,2}$, Ho Leung Ng Corresp. 1,3 \\ ${ }^{1}$ Department of Chemistry, University of Hawaii at Manoa, Honolulu, Hawaii, United States of America \\ 2 School of Medicine, St. Louis University, St. Louis, Missouri, United States of America \\ 3 University of Hawaii Cancer Center, Honolulu, Hawaii, United States of America \\ Corresponding Author: Ho Leung $\mathrm{Ng}$ \\ Email address: hng@hawaii.edu
}

Fibroblast growth factor receptors (FGFRs) are a family of receptor tyrosine kinases containing three domains: an extracellular receptor domain, a single transmembrane helix, and an intracellular tyrosine kinase domain. FGFRs are activated by fibroblast growth factors (FGFs) as part of complex signal transduction cascades regulating angiogenesis, skeletal formation, cell differentiation, proliferation, cell survival, and cancer. We have developed the first recombinant expression system in E. coli to produce a construct of human FGFR2 containing its transmembrane and extracellular receptor domains. We demonstrate that the expressed construct is functional in binding heparin and dimerizing. Size exclusion chromatography demonstrates that the purified FGFR2 does not form a complex with FGF1 or adopts an inactive dimer conformation. Progress towards the successful recombinant production of intact FGFRs will facilitate further biochemical experiments and structure determination that will provide insight into how extracellular FGF binding activates intracellular kinase activity. 
1 TITLE: Recombinant expression in E. coli of human FGFR2 with its transmembrane and

2 extracellular domains

3

4 Authors: Adam Bajinting ${ }^{1,2}$, Ho Leung Ng ${ }^{1,3}$

$5{ }^{1}$ Department of Chemistry, University of Hawaii at Manoa

$6{ }^{2}$ St. Louis University, School of Medicine

$7 \quad{ }^{3}$ University of Hawaii Cancer Center

8

9 Corresponding author: Ho Leung Ng, hng@hawaii.edu

11 ABSTRACT

12 Fibroblast growth factor receptors (FGFRs) are a family of receptor tyrosine kinases

13 containing three domains: an extracellular receptor domain, a single transmembrane helix, and an intracellular tyrosine kinase domain. FGFRs are activated by fibroblast growth factors (FGFs) as part of complex signal transduction cascades regulating angiogenesis, skeletal formation, cell differentiation, proliferation, cell survival, and cancer. We have developed the first recombinant expression system in E. coli to produce a construct of human FGFR2 containing its transmembrane and extracellular receptor domains. We demonstrate that the expressed construct is functional in binding heparin and dimerizing. Size exclusion chromatography demonstrates that the purified FGFR2 does not form a complex with FGF1 or adopts an inactive dimer conformation. Progress towards the successful recombinant production of intact FGFRs will

22 facilitate further biochemical experiments and structure determination that will provide insight into how extracellular FGF binding activates intracellular kinase activity. 
27

\section{INTRODUCTION}

As receptor tyrosine kinases (RTKs), FGFRs have three primary domains: an extracellular domain (ECD), a single transmembrane helix (TM), and an intracellular tyrosine kinase domain (KD) (Fig. 1). These proteins are expressed primarily in endothelial, fibroblast, vascular smooth muscle, neuroectodermal, and mesenchymal cells. When activated by fibroblast growth factors (FGFs), these receptors are responsible for activating mechanisms via transautophosphorylation that result in angiogenesis, skeletal formation, and cell differentiation, proliferation, survival, and growth. Within the subfamily are four types of FGFRs: FGFR1, FGFR2, FGFR3, and FGFR4, which share 55-72\% sequence homology. Due to their critical roles in cell and tissue development, mutations of FGFRs are known to lead to achondroplasia (poor cartilage growth) and developmental disorders that exhibit craniosynostosis (improper skull formation) (Turner \& Grose, 2010). FGFR2 and FGFR3 have also been implicated in cancers such as bladder cancer, and inhibitors are being investigated as potential cancer therapeutics (Turner \& Grose, 2010; Brooks, Kilgour \& Smith, 2012; Daniele et al., 2012; Dieci et al., 2013).

ECD

TM

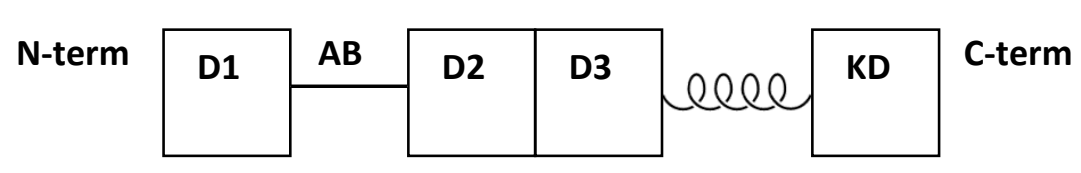

Fig. 1. Schematic of domains and motifs in FGFR2. D1-D3 are the immunoglobulin domains. $\mathrm{AB}$ is the acid box motif. ECD is the extracellular domain (or ectodomain). TM is the single transmembrane helix. KD is the intracellular kinase domain. 

immunoglobulin (Ig) domains termed D1, D2, and D3. Between D1 and D2 is an acid box motif, a sequence of 20 acid-rich amino acids that binds to divalent cations to stabilize the interaction between FGFR and heparin/heparin sulfate proteoglycans (HSPGs) (Patstone \& Maher, 1996). The acid box also mediates interactions with other proteins (Sanchez-Heras et al., 2006, p.) and plays a key role in auto-inhibition (Kalinina et al., 2012). For the FGFR2 ECD+TM construct in particular, the structure of the ECD lacks both the acid box and the D3 domain. Removal of both regions increases the affinity for heparin and the ability of FGF to active FGFR (Wang et al., 1995). The D3 domain is unnecessary for FGF1 activation and is involved in differential responses to different FGFs (Yu et al., 2000). transmembrane helix and how it connects the ECD and KD. There is an NMR structure of the FGFR3 TM that shows it as a single alpha helix (Bocharov et al., 2013). However, the biological relevance of this structure is unclear as the data was collected from a construct containing only the TM and the extracellular juxtamembrane region, without the ECD or KD. As the TM represents a tiny proportion of the full-length FGFR, it is likely that the natural conformation of the TM in the intact receptor in vivo differs significantly from the isolated peptide. 

more insight into the receptor activation mechanism and how activation status is transduced

across the membrane. Bocharov et al. proposed a "string puppet theory" mechanism of signal transduction based on the NMR structure of the TM helix (Bocharov et al., 2013). The string puppet theory proposes that FGFR dimerizes in an inactive form via its transmembrane domains without FGF and heparin; the active conformation results when the inactive dimer binds to FGFs. Details of the stoichiometry of FGF, heparin, and FGFR in the activated complex are also debated (Lemmon \& Schlessinger, 2010). Conclusively resolving different hypotheses about inactive and active FGFR states will come from detailed structures of intact FGFR.

Here we describe our development of a recombinant expression system in E. coli to produce significant quantities of functional FGFR with its TM linked to either its ECD or KD for eventual structural studies. Recombinant expression of complex eukaryotic proteins in E. coli is often challenging and results in low yields of insoluble, inactive protein (Rosano \& Ceccarelli, 2014). Expression of membrane proteins containing the very hydrophobic transmembrane domains is especially problematic (Hattab et al., 2015). Moreover, there have been only a few studies describing the successful heterologous expression of protein kinases including their transmembrane domains, with none expressed in E. coli (Mi et al., 2008, 2011; Lu et al., 2012; Paavilainen et al., 2013; Opatowsky et al., 2014; Chen, Unger \& He, 2015). These prior studies describe the recombinant expression of EGFR, EphA2, PDGFR, and Kit. Here, we describe the expression of constructs of FGFR2 and FGFR3 containing ECD+TM in E. coli in sufficient yield for protein crystallization. FGFR was expressed as a fusion protein with maltose binding protein (MBP), which has been shown to improve expression yield and solubility (Kapust \& Waugh, 1999). We show that the FGFR2 ECD+TM construct is functional in binding heparin and 
92 dimerizing. Our simple recombinant method will facilitate biochemical experiments studying the

93 relationship between the TM and other domains.

\section{MATERIALS \& METHODS}

96

97

98

99

100

101

102

103

104

105

106

107

108

109

\section{DNA cloning of constructs}

PIPE (polymerase incomplete primer extension) cloning was used to obtain specific domain combinations of FGFR2, and the cloning vector pSpeedET with an N-terminal E. coli maltose binding protein (MBP) fusion tag of $42.5 \mathrm{kDa}$ (Klock \& Lesley, 2009). The domain combinations created are shown in Table 1. The FGFR inserts were amplified by PCR using Phusion Hi Fidelity DNA Polymerase, $200 \mathrm{mM}$ dNTP, $0.5 \mu \mathrm{M}$ forward and reverse primers, and 6\% DMSO. PCR products were extracted from agarose gel and purified using Thermo Scientific GeneJet Gel Extraction Kits. The MBP fusion tag was added to the construct to improve construct solubility and expression (Kapust \& Waugh, 1999), allow purification by amylose affinity chromatography, and identification by Western blot with an anti-MBP antibody (New England Biolabs (E-8038)). Cloning results were confirmed by DNA sequencing.

\section{Table 1. FGFR2 and FGFR3 constructs created}

\begin{tabular}{|l|l|}
\hline Construct & Expected Size (kDa) \\
\hline MBP + FGFR2 31-406 (ECD + TM) & 71.5 \\
\hline MBP + FGFR2 370-651 (TM + KD) & 73.7 \\
\hline MBP + FGFR2 31-651 (ECD + TM + KD) & 111.5 \\
\hline
\end{tabular}




\begin{tabular}{|l|l|}
\hline MBP + FGFR3 143-405 (ECD + TM) & 71.3 \\
\hline MBP + FGFR3 365-771 (TM + KD) & 87.9 \\
\hline MBP + FGFR3 143-771 (ECD + TM + KD) & 112.3 \\
\hline
\end{tabular}

110

111

112

113

114

115

116

117

118

119

120

121

122

123

124

125

126

127

128

129

\section{Small-scale expression}

Small scale expression studies were performed using E. coli Lemo21 cells (New England Biolabs). $10 \mathrm{~mL}$ inoculate from an overnight culture was added to $100 \mathrm{~mL}$ of TB media and shaken at $37^{\circ} \mathrm{C}$. The $\mathrm{OD}_{600}$ was monitored as it approached an absorbance of 0.6 . Once the culture reached an $\mathrm{OD}_{600}$ of $0.4-0.5$, the cells were cooled to $18^{\circ} \mathrm{C}$ in the shaker to slow the growth of cells and $0.5 \mathrm{mM}$ rhamnose was added to a final concentration of $0.5 \mathrm{mM}$ to titrate expression levels in the Lemo21 cells. Once it reached OD of $0.6,1 \mathrm{~mL}$ of each construct culture was taken to serve as a negative control for later experiments. Isopropyl $\beta$-D-1thiogalactopyranoside (IPTG) was then added at a $0.1 \mathrm{mM}$ final concentration to each culture to induce expression. The cells were then grown in a shaker at $18^{\circ} \mathrm{C}$ overnight.

\section{Harvesting and lysing cells}

Each of the cultures was centrifuged at $4^{\circ} \mathrm{C}$ at $4,800 \mathrm{~g}$ for 10 minutes. The culture media was discarded, and the pellet was washed by resuspending in lysis buffer $(300 \mathrm{mM} \mathrm{NaCl}, 50 \mathrm{mM}$ HEPES at $\mathrm{pH} 7.5,0.1 \mathrm{mM} \mathrm{MgSO}_{4}, 5 \%$ glycerol, $0.5 \mathrm{mM}$ TCEP, benzamidine, and PMSF). It was centrifuged at 4,800 $\mathrm{g}$ for 10 minutes, after which, the lysis buffer was discarded. $20 \mathrm{mg}$ of post induction $E$. coli cell pellet was resuspended in $180 \mu \mathrm{L}$ of lysis buffer $(300 \mathrm{mM} \mathrm{NaCl}, 50$ mM HEPES at pH 7.5, 0.1 mM MgSO, $5 \%$ glycerol, $0.5 \mathrm{mM}$ TCEP, benzamidine, and PMSF). 
$13020 \mu \mathrm{L}$ of $10 \mathrm{mg} / \mathrm{mL}$ lysozyme stock was added in addition to $0.3 \mu \mathrm{L}$ of DNAse I. Next, the lysis

131 reaction was put through three freeze-thaw cycles to lyse the cells.

132

133

134

135

136

137

138

139

140

141

142

143

144

145

146

147

148

149

150

151

152

153

\section{Western blot analysis}

Western blotting was performed on PVDF membranes after wet transfer from polyacrylamide gels. Membranes were blocked with Amresco RapidBlock solution for 5 minutes and then incubated with HRP-conjugated anti-MBP monoclonal antibody (New England Biolabs) overnight at $4^{\circ} \mathrm{C}$. Membranes were then washed three times for 5 minutes with $20 \mathrm{mM}$ Tris- $\mathrm{HCl} \mathrm{pH} 7.5,150 \mathrm{mM} \mathrm{NaCl}$, and 0.1\% Tween 20. Finally, the blots were developed using the KPL TMB Membrane Peroxidase Substrate System kit.

\section{Large-scale expression studies}

After the best candidates for continued expression studies were determined, the FGFR2 ECD+TM constructs were expressed at a larger scale. The expression procedures (transformation and inoculation) are identical except that instead of $10 \mathrm{~mL}$ of initial culture (in LB) to inoculate $100 \mathrm{~mL}$ of $\mathrm{TB}, 100 \mathrm{~mL}$ of initial culture was grown and inoculated into 1000 $\mathrm{mL}$ of $\mathrm{TB}$.

Once the culture reached $\mathrm{OD}_{600}$ of $0.4-0.5$, the cells were cooled to $18^{\circ} \mathrm{C}$ in the shaker to slow growth, and rhamnose was added to a final concentration of $0.5 \mathrm{mM}$ to titrate expression levels in the Lemo21 cells. Isopropyl $\beta$-D-1-thiogalactopyranoside (IPTG) was then added at 0.1 $\mathrm{mM}$ final concentration to induce expression.

\section{Cell lysis}


156 final concentration of $\mathrm{CaCl}_{2}$, and additional protease inhibitors (E-64, pepstatin, and bestatin)

157 prior to lysis by sonication at $4^{\circ} \mathrm{C}$ with a Fisher Scientific P-550. Sonication was performed for

158 a total of 2 minutes, divided into 20 seconds of sonication followed by 40 seconds of rest (total

159 of 6 minutes of clock time), at $60 \%$ of full power. Samples are kept on ice during sonication.

160 After sonication, the suspensions were centrifuged at 48,400 g for 30 minutes.

\section{Detergent extraction of FGFR from cell membranes}

Unlike the small-scale expression trials, large-scale expression studies included detergent

supernatant, $500 \mu \mathrm{L}$ of lysis buffer with $1 \%$ detergent solution was added and resuspended in the presence of PMSF. The suspension for each was then constantly inverted for 2 hours at $25^{\circ} \mathrm{C}$. The suspensions were then centrifuged at 20,800 g. Both pellet and supernatant were then stored at $-80^{\circ} \mathrm{C}$. Several detergents were tested for optimal extraction from the cell pellet and the supernatant from the centrifugation: $1 \%$ n-dodecyl- $\beta$-D-maltopyranoside (DDM), 1\% Brij 35, and $1 \%$ Brij 58 for the samples of pellet and supernatant. FGFR2/3 constructs were tested for binding to MBP-Trap HP affinity chromatography resin (GE Healthcare).

\section{Refolding by dialysis}

Both FGFR2 and FGFR3 constructs containing ECD+TM were refolded by dialysis as washed and resuspended with $0.5 \%$ guanidinium- $\mathrm{HCl}$ and centrifuged at $45,000 \mathrm{~g}$ for 20 
177 minutes. Next, the pellets were solubilized in dialysis solution \#1 (6 M guanidinium-HCl, 0.1\%

178 DDM, 10 mM DTT, and protease inhibitors E-64, benzamidine, PMSF, bestatin, and pepstatin at 179 a $\mathrm{pH}$ of 8.0). To facilitate solubilization, the cell pellet and dialysis solution mixture was warmed 180 briefly to $40^{\circ} \mathrm{C}$ and then vortexed at room temperature. The total mixture was about $13 \mathrm{~mL}$. All $18113 \mathrm{~mL}$ of the solubilized inclusion bodies in the dialysis solution \#1 was loaded into a dialysis 182 membrane. This was placed in a beaker with $700 \mathrm{~mL}$ of dialysis solution \#2 (25 mM HEPES, $183150 \mathrm{mM} \mathrm{NaCl}, 10 \%$ glycerol, and $1 \mathrm{mM}$ L-cysteine at $\mathrm{pH} 7.5)$ at $4^{\circ} \mathrm{C}$ overnight with constant 184 stirring using a magnetic stir bar. After 19 hours, the sample within the dialysis membrane was then centrifuged at $24,000 \mathrm{~g}$ for 30 minutes and the supernatant was stored at $-80^{\circ} \mathrm{C}$.

\section{FGF1 expression and purification}

The FGF1 gene with an N-terminal His-tag in the expression vector pMCSG7 was obtained from the DNASU Plasmid Repository at Arizona State University. FGF1 was first purified using a $1 \mathrm{~mL}$ HiTrap GE Healthcare heparin affinity chromatography column, using elution buffer containing $1 \mathrm{M} \mathrm{NaCl}, 10 \%$ glycerol, $25 \mathrm{mM}$ HEPES, $10 \mathrm{mM}$ imidazole, and benzamidine with a $\mathrm{pH}$ of 7.5 , as described previously (Pellegrini et al., 2000).

PAGE and Western blot analysis, but instead of using an anti-MBP antibody (HRP conjugated), an anti-His antibody (HRP conjugated) from Pierce was used. This was then followed by size exclusion chromatography on a Superdex 200 10/300 GL column (GE Healthcare). The running $\mathrm{mM} \mathrm{NaCl}$. 
200

201

202

203

204

205

206

207

208

209

210

211

212

213

214

215

216

217

218

219

220

221

222

\section{Initial functionality test of FGFR2}

The first step in testing functionality is to determine whether FGFR2 can bind to heparin and FGF1. The $1 \mathrm{~mL}$ HiTrap Heparin Affinity Chromatography (GE Healthcare) column was used to test for heparin binding. To equilibrate the column, 10 column volumes (CVs) of binding buffer (150 mM NaCl, 25 mM HEPES pH 7.5, benzamidine, and 0.1\% DDM) was loaded with a syringe. Next $1 \mathrm{~mL}$ of FGF1's elution fraction from SEC was loaded onto the column and then 10 CVs of binding buffer. This was followed by FGFR2's supernatant from the dialysis. After loading, FGFR2 was washed with 5 CVs of binding buffer and eluted with 10 CVs of elution buffer (25 mM HEPES pH 7.5, $1.5 \mathrm{M} \mathrm{NaCl}, 0.1 \%$ DDM, benzamidine, and PMSF).

After chromatography, SDS-PAGE and western blot analysis were performed. The antiMBP antibody was used to detect FGFR2, and the anti-His-tag antibody was used to detect FGF1. After heparin affinity chromatography, we performed size exclusion chromatography to assess the presence of aggregation, suggesting non-functional protein, or dimers, supporting functional protein.

\section{RESULTS}

\section{Small-scale expression of FGFR2 and FGFR3 constructs}

We performed small-scale expression trials of the FGFR2 and FGFR3 constructs in 100 $\mathrm{mL}$ of culture volume to determine which TM-containing construct would likely produce the highest yield for larger scale expression studies. Initial expression trials of FGFRs in the Rosetta2(DE3) strain of $E$. coli demonstrated extensive cell death after IPTG induction, suggesting toxicity of the expressed protein. We were able to express FGFRs and avoid expression toxicity using $E$. coli strain Lemo21, that contains T7 RNA polymerase that is 
223 titratable by rhamnose added to the media, a feature useful for expressing poorly folding

224 membrane proteins and toxic proteins (Wagner et al., 2008; Schlegel et al., 2012). The western

225 blot with an anti-MBP antibody showed significant quantities of FGFR2 and FGFR3 ECD+TM

226 in both the soluble and cell pellet fractions (Fig. 2, lanes 2, 4, 7, 9). The intact receptors were not

227 detected (data not shown). FGFR2 TM+KD was not detected (lanes 3,8), but FGFR3 TM+KD

228 (lane 10) was found in the cell pellet fraction. We considered the FGFR2 and FGFR3 ECD+TM

229 constructs (lanes 7 and 9) to be the most promising for larger scale expression studies because of

230 their superior yield, and the partial recovery of soluble FGFR3 ECD+TM (lane 4). In addition,

231 for most of the constructs, prominent bands corresponding to the molecular weight of MBP were

232 observed suggesting significant proteolysis of the fusion protein. This was not considered

233 problematic as eventual structural studies would require removal of the MBP fusion-tag

234 downstream of purification.

235

236

237

238

239

240

241

242

243

244

245

246

247

248

249

250

251

252

253 $\begin{array}{llllllllll}1 & 2 & 3 & 4 & 5 & 6 & 7 & 8 & 9 & 10\end{array}$

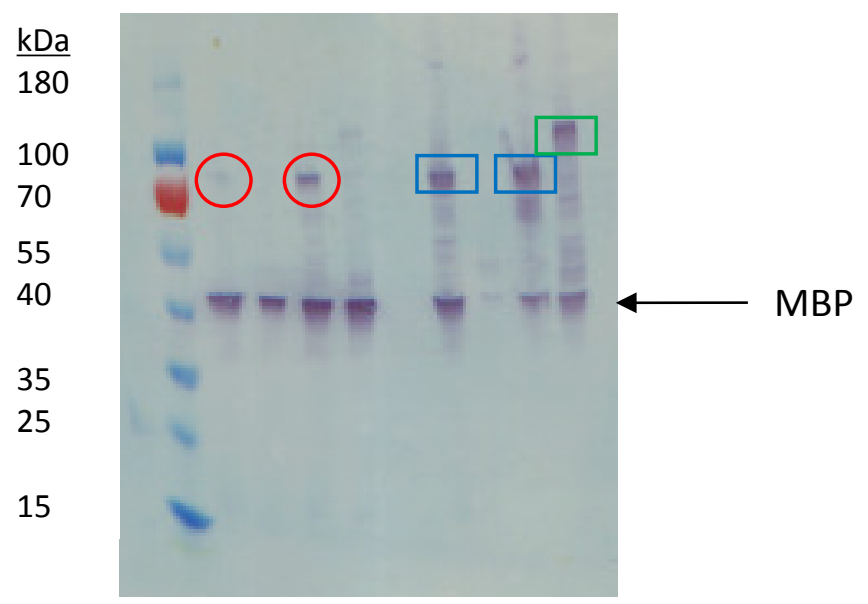

Fig. 2. Western blot analysis of small-scale expression of FGFR2 and FGFR3 constructs using anti-MBP antibody. Lane 1: Ladder. Lane 2: FGFR2 31-406 from supernatant. Lane 3: FGFR2 370-651 from supernatant. Lane 4: FGFR3 143-405 from supernatant. Lane 5: FGFR 3: 365-771 from supernatant. Lane 6: Blank. Lane 7: FGFR2 31-406 from pellet. Lane 8: FGFR2 370-651 from pellet. Lane 9: FGFR3 143-405 from pellet. Lane 10: FGFR3 365-771 from pellet. Circled in red are bands consistent with FGFR2 and FGFR3 ECD+TM from supernatant. Boxed 
254 in blue are bands consistent with FGFR 2 and 3 ECD+TM from the cell pellet fraction. Boxed in 255 green is a band consistent with FGFR3 TM+KD from the cell pellet fraction.

256

257

258

259

260

261

262

263

264

265

266

267

268

269

270

271

272

273

274

275

276

277

\section{Large-scale expression studies and detergent extraction analysis}

After determining the optimal constructs from the small-scale expression studies, we performed expression trials of the FGFR2 and FGFR3 ECD+TM constructs in larger scale, 1L cultures of Lemo 21 cells. We tested three detergent solutions, containing $1 \% \mathrm{n}$-dodecyl- $\beta$-Dmaltopyranoside (DDM), Brij 35, or Brij 58, for extraction of FGFR2/3 from cells. The western blot with an anti-MBP antibody on the detergent-extracted fractions showed significant quantities of FGFR2 and FGFR3 ECD+TM from both the soluble and cell pellet fractions (Fig. 3). We determined that DDM (lanes 2, 3, 8, and 9), Brij 35 (lanes 4, 5, 10, and 11), and Brij 58 (lanes 6, 7, 12, and 13) extracted FGFR2/3 to similar levels. We selected DDM for all subsequent procedures because it is the most commonly used detergent for membrane protein crystallography (Privé, 2007; Loll, 2014). As in the small-scale expression trials, we observed prominent bands corresponding to proteolyzed MBP in the western blots. Due to the large amounts of protein loaded, we also observed high amounts of non-specific binding in the western blot. We also observed a high molecular weight band that comigrated near the $180 \mathrm{kDa}$ ladder band that we tentatively identify as oligomerized or aggregated FGFR2/3.

Based on the high expression levels shown on this western blot, especially from the soluble fraction, we initially decided FGFR3 ECD+TM would be our lead candidate for further expression and purification studies. However, we found that FGFR3 from the soluble fraction did not bind to the MBP affinity column. This suggested that the fusion protein, MBP-FGFR3 ECD+TM, was folded incorrectly, despite being soluble. Thus, we refocused efforts on recovering FGFR2 from the insoluble fractions. We pursued expression of FGFR2 ECD+TM in 
278 inclusion bodies and refolding by dialysis, as demonstrated previously for FGFR2 ECD

279 (Mohammadi, Schlessinger \& Hubbard, 1996). Refolding provided high yields of FGFR2

$280 \mathrm{ECD}+\mathrm{TM},>4 \mathrm{mg}$ of purified protein from $1 \mathrm{~L}$ of culture. The yield is adequate for protein

281 crystallization.

282

283

284

285

286

287

288

289

290

291

292

293

294

295

296

297

298

299

300

301

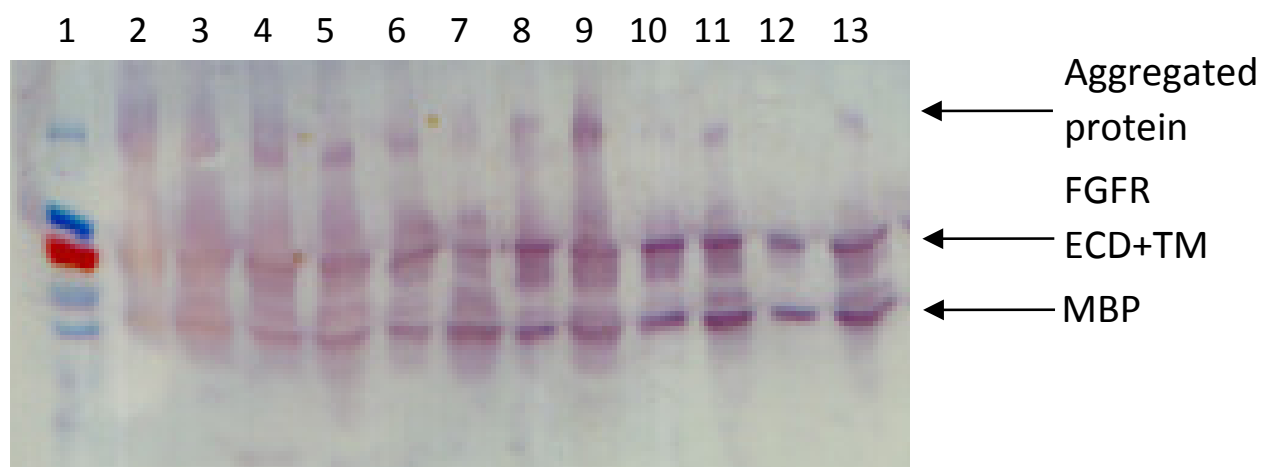

Fig. 3. Western blot of detergent extractions of large-scale expression constructs FGFR2 and FGFR3 ECD+TM. Lane 1: Ladder. Lane 2: FGFR2 pellet with 1\% DDM. Lane 3: FGFR 2 supernatant with 1\% DDM. Lane 4: FGFR2 pellet with 1\% Brij 35. Lane 5: FGFR2 supernatant with 1\% Brij 35. Lane 6: FGFR2 pellet with 1\% Brij 58. Lane 7: FGFR2 supernatant with 1\% Brij 58. Lane 8: FGFR3 pellet with 1\% DDM. Lane 9: FGFR3 supernatant with 1\% DDM. Lane 10: FGFR3 pellet with 1\% Brij 35. Lane 11: FGFR3 supernatant with Brij 35. Lane 12: FGFR3 pellet with 1\% Brij 58. Lane 13: FGFR3 supernatant with 1\% Brij 58.

\section{Binding of refolded FGFR2 to heparin}

To test that the refolded FGFR2 ECD+TM retained its function, we sought to determine whether it could 1) bind heparin, 2) bind FGF1, and 3) dimerize. We tested the refolded fraction for binding to a heparin affinity chromatography column. A western blot with an anti-MBP antibody of the eluted fractions from the heparin affinity column demonstrated the presence of MBP-FGFR2 ECD+TM, supporting that the refolded FGFR2 bound heparin (Fig. 4). 
302

303

304

305

306

307

308

309

310

311

312

313

314

315

316

317

318

319

320

321

322

323 shown).

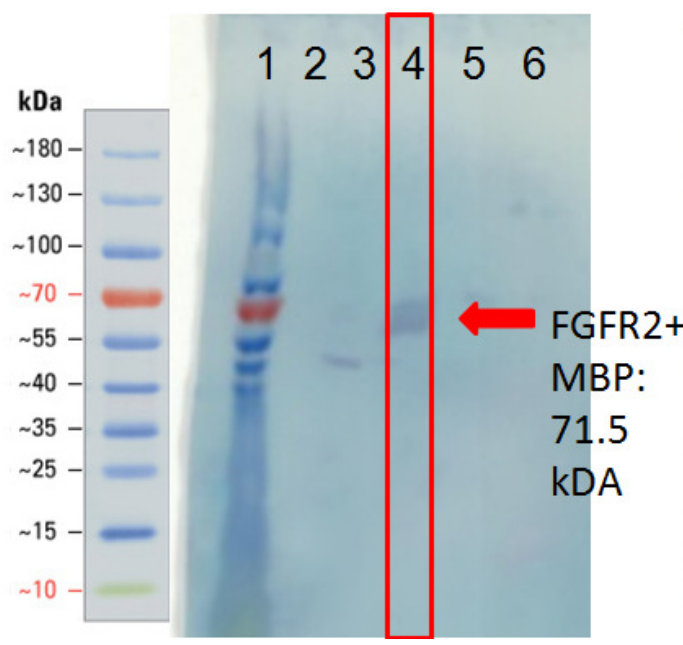

Fig. 4. Western blot of heparin affinity column purification fractions using anti-MBP antibody. Lane 1: ladder. Lanes 2-3: wash fractions. Lanes 46: elution fractions.

\section{Refolded FGFR2 forms dimers but does not bind FGF1}

The main elution fraction from the heparin affinity purification was then passed through a size exclusion chromatography column to resolve its components (Fig. 5). The first peak, eluting at $25.76 \mathrm{~mL}$, corresponds to a molecular weight of $200 \mathrm{kDa}$. The second peak, eluting at 30.79 $\mathrm{mL}$, corresponds to between 66 and $79 \mathrm{kDa}$. The third primary peak, eluting at $34.99 \mathrm{~mL}$, corresponds to a size between 12 and $20 \mathrm{kDa}$. Each of these three primary peaks was analyzed by SDS-PAGE and western blots (Fig. 6). The second peak corresponded to the molecular weight of the MBP-FGFR2 ECD+TM construct $(71.5 \mathrm{kDa})$ and was identified by western blot with an anti-MBP antibody (data not shown). The third peak corresponded to FGF1 from its molecular weight $(17.5 \mathrm{kDa})$ and was identified by western blot with an anti-His-tag antibody (data not 


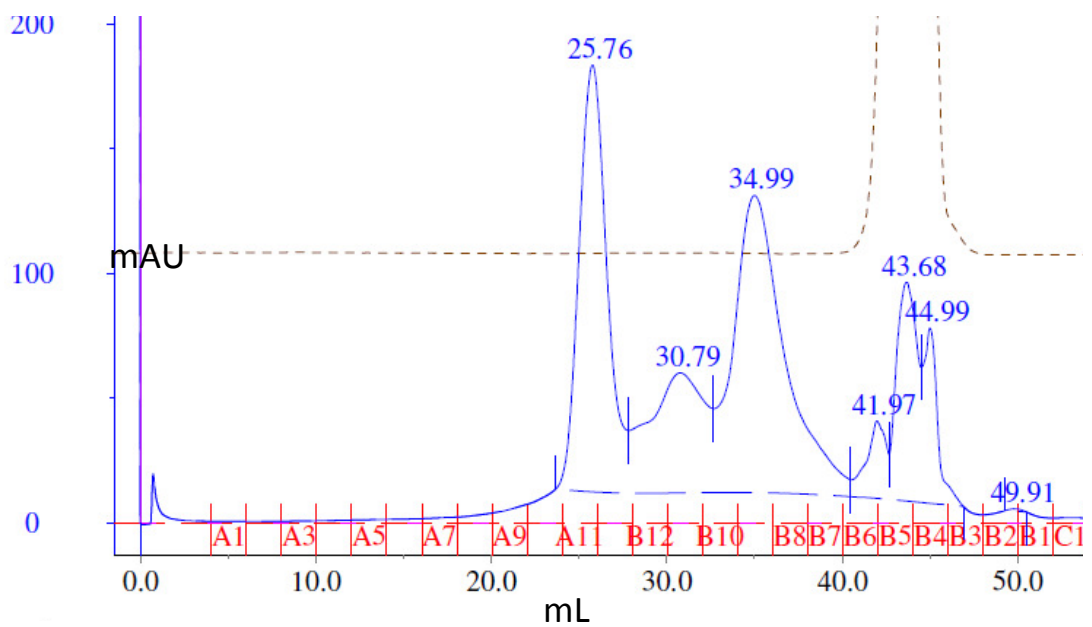

Fig. 5. Size exclusion chromatography of the main heparin affinity elution fraction.

We considered two possibilities for the identity of the first peak: 1) a complex of MBP-

FGFR2 dimer with FGF1, 2) a dimer of MBP-FGFR2 in DDM micelles. Western blot analysis

337

338

339

340

341

342

343 344

345

346

347

348

349 with an anti-MBP antibody confirmed the presence of MBP-FGFR2 ECD+TM (Fig. 6). We eliminated the possibility of the peak being the FGFR2-FGF1 complex because the expected molecular weight is $230.5 \mathrm{kDa}$, and western blot analysis with an anti-His-tag antibody did not show the presence of FGF1 (data not shown). In contrast, the expected molecular weight of an MBP-FGFR ECD-TM dimer with a DDM micelle is $213 \mathrm{kDa}$. Potentially, the inclusion of the TM region or DDM may stabilize an inactive conformation of the dimer, rendering it incapable of binding FGF1. Another possibility is that the MBP fusion-tag interfered with FGF1 binding. 
350

351

352

353

354

355

356

357

358

359

360

361

362

363

364

365

366

367

368

369

370

371

372

373
12

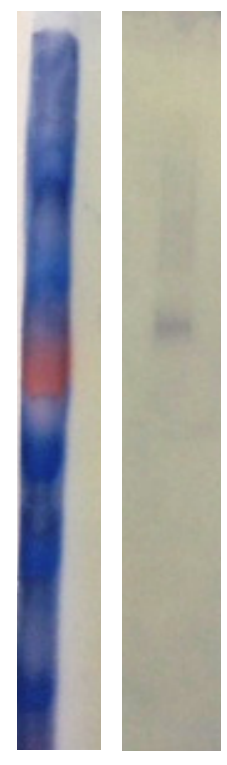

MBP-FGFR2 ECD+TM

Fig. 6. Western blot of the first peak from SEC using anti-MBP antibody. Arrow points to band correlating to FGFR2 construct size (71.5kDa). Lane 1: Ladder. Lane 2: SEC fraction A12. Both lanes are cropped from the same blot.

\section{CONCLUSIONS}

Our results present progress toward recombinantly expressing partially functional FGFR2 ECD+TM in E. coli. This is the first report of recombinant expression in E. coli of a eukaryotic protein kinase construct containing its TM domain. Protein production in E. coli is highly desirable because of low costs, fast growth, easy mutagenesis, and high protein yields. Key steps include the use of the MBP fusion tag, use of the Lemo21 (DE3) strain, refolding from inclusion bodies, and use of the detergent DDM throughout all extraction and purification procedures. The purified FGFR2 ECD+TM demonstrated the ability to dimerize and bind heparin but did not form a stable complex with FGF1 as observed by size exclusion chromatography. This may suggest that the purified FGFR2 was not fully folded or functional. Other possible explanations include 1) inclusion of the TM or detergent favors an inactive conformation, 2) stable complex formation requires the addition of accessory molecules such as heparin, heparan sulfate, or sodium octasulfate (Zhang et al., 2009), or 3) the MBP fusion tag interfered with FGF1 binding. We plan future experiments to address these possibilities. The potential inhibitory role of the TM 
374 in FGF binding and receptor activation merits further investigation. Future studies of FGFR and

375 receptor kinase function should include the TMs in the expressed protein constructs as its

376 biochemical role is increasingly recognized.

377

378

379

380

381

382

383

384

385

386

387

388

389

390

391

392

393

394

395

396

\section{REFERENCES}

Bocharov EV., Lesovoy DM., Goncharuk SA., Goncharuk MV., Hristova K., Arseniev AS. 2013. Structure of FGFR3 Transmembrane Domain Dimer: Implications for Signaling and Human Pathologies. Structure 21:2087-2093. DOI: 10.1016/j.str.2013.08.026.

Brooks AN., Kilgour E., Smith PD. 2012. Molecular Pathways: Fibroblast Growth Factor Signaling: A New Therapeutic Opportunity in Cancer. Clinical Cancer Research 18:1855-1862. DOI: 10.1158/1078-0432.CCR-11-0699.

Chen P-H., Unger V., He X. 2015. Structure of Full-Length Human PDGFR $\beta$ Bound to Its Activating Ligand PDGF-B as Determined by Negative-Stain Electron Microscopy. Journal of Molecular Biology 427:3921-3934. DOI: 10.1016/j.jmb.2015.10.003.

Daniele G., Corral J., Molife LR., Bono JS de. 2012. FGF Receptor Inhibitors: Role in Cancer Therapy. Current Oncology Reports 14:111-119. DOI: 10.1007/s11912-012-0225-0.

Dieci MV., Arnedos M., Andre F., Soria JC. 2013. Fibroblast Growth Factor Receptor Inhibitors as a Cancer Treatment: From a Biologic Rationale to Medical Perspectives. Cancer Discovery 3:264-279. DOI: 10.1158/2159-8290.CD-12-0362.

Hattab G., Warschawski DE., Moncoq K., Miroux B. 2015. Escherichia coli as host for membrane protein structure determination: a global analysis. Scientific Reports 5:12097. DOI: $10.1038 /$ srep12097. 
397 Kalinina J., Dutta K., Ilghari D., Beenken A., Goetz R., Eliseenkova AV., Cowburn D.,

398 Mohammadi M. 2012. The alternatively spliced acid box region plays a key role in FGF receptor autoinhibition. Structure 20:77-88. DOI: 10.1016/j.str.2011.10.022.

400 Kapust RB., Waugh DS. 1999. Escherichia coli maltose-binding protein is uncommonly

401

402 effective at promoting the solubility of polypeptides to which it is fused. Protein Science

403

404

405

406

407

408

409

410

411

412

413

414

415

416

417

418

419 8:1668-1674. DOI: $10.1110 /$ ps.8.8.1668.

Klock HE., Lesley SA. 2009. The Polymerase Incomplete Primer Extension (PIPE) method applied to high-throughput cloning and site-directed mutagenesis. Methods in Molecular Biology 498:91-103. DOI: 10.1007/978-1-59745-196-3_6.

Lemmon MA., Schlessinger J. 2010. Cell Signaling by Receptor Tyrosine Kinases. Cell 141:1117-1134. DOI: 10.1016/j.cell.2010.06.011.

Loll PJ. 2014. Membrane proteins, detergents and crystals: what is the state of the art? Acta Crystallographica Section F Structural Biology Communications 70:1576-1583. DOI: $10.1107 /$ S2053230X14025035.

Lu C., Mi L-Z., Schürpf T., Walz T., Springer TA. 2012. Mechanisms for Kinase-mediated Dimerization of the Epidermal Growth Factor Receptor. Journal of Biological Chemistry 287:38244-38253. DOI: 10.1074/jbc.M112.414391.

Mi L-Z., Grey MJ., Nishida N., Walz T., Lu C., Springer TA. 2008. Functional and Structural Stability of the Epidermal Growth Factor Receptor in Detergent Micelles and Phospholipid Nanodiscs†. Biochemistry 47:10314-10323. DOI: 10.1021/bi801006s.

Mi L-Z., Lu C., Li Z., Nishida N., Walz T., Springer TA. 2011. Simultaneous visualization of the extracellular and cytoplasmic domains of the epidermal growth factor receptor. Nature Structural \& Molecular Biology 18:984-989. DOI: 10.1038/nsmb.2092. 
420 Mohammadi M., Schlessinger J., Hubbard SR. 1996. Structure of the FGF receptor tyrosine $421 \quad$ kinase domain reveals a novel autoinhibitory mechanism. Cell 86:577-587.

422 Opatowsky Y., Lax I., Tomé F., Bleichert F., Unger VM., Schlessinger J. 2014. Structure, 423 domain organization, and different conformational states of stem cell factor-induced intact KIT dimers. Proceedings of the National Academy of Sciences 111:1772-1777. DOI: 10.1073/pnas.1323254111.

426

427

428

429

430

431

432

433

434

435

436

437

438

439

440

441

Paavilainen S., Grandy D., Karelehto E., Chang E., Susi P., Erdjument-Bromage H., Nikolov D., Himanen J. 2013. High-level expression of a full-length Eph receptor. Protein Expression and Purification 92:112-118. DOI: 10.1016/j.pep.2013.08.016.

Patstone G., Maher P. 1996. Copper and calcium binding motifs in the extracellular domains of fibroblast growth factor receptors. Journal of Biological Chemistry 271:3343-3346.

Pellegrini L., Burke DF., von Delft F., Mulloy B., Blundell TL. 2000. Crystal structure of fibroblast growth factor receptor ectodomain bound to ligand and heparin. Nature 407:1029-1034. DOI: 10.1038/35039551.

Plotnikov AN., Hubbard SR., Schlessinger J., Mohammadi M. 2000. Crystal structures of two FGF-FGFR complexes reveal the determinants of ligand-receptor specificity. Cell $101: 413-424$.

Plotnikov AN., Schlessinger J., Hubbard SR., Mohammadi M. 1999. Structural Basis for FGF Receptor Dimerization and Activation. Cell 98:641-650. DOI: 10.1016/S00928674(00)80051-3.

Privé GG. 2007. Detergents for the stabilization and crystallization of membrane proteins. Methods 41:388-397. DOI: 10.1016/j.ymeth.2007.01.007. 
442 Rosano GL., Ceccarelli EA. 2014. Recombinant protein expression in Escherichia coli: advances and challenges. Frontiers in Microbiology 5:172. DOI: 10.3389/fmicb.2014.00172.

444 Sanchez-Heras E., Howell FV., Williams G., Doherty P. 2006. The fibroblast growth factor

445

446

447

448

449

450

451

452

453

454

455

456

457

458

459

460

461

462

463

receptor acid box is essential for interactions with $\mathrm{N}$-cadherin and all of the major isoforms of neural cell adhesion molecule. Journal of Biological Chemistry 281:3520835216. DOI: 10.1074/jbc.M608655200.

Schlegel S., Löfblom J., Lee C., Hjelm A., Klepsch M., Strous M., Drew D., Slotboom DJ., de Gier J-W. 2012. Optimizing Membrane Protein Overexpression in the Escherichia coli strain Lemo21(DE3). Journal of Molecular Biology 423:648-659. DOI: 10.1016/j.jmb.2012.07.019.

Schlessinger J., Plotnikov AN., Ibrahimi OA., Eliseenkova AV., Yeh BK., Yayon A., Linhardt RJ., Mohammadi M. 2000. Crystal Structure of a Ternary FGF-FGFR-Heparin Complex Reveals a Dual Role for Heparin in FGFR Binding and Dimerization. Molecular Cell 6:743-750. DOI: 10.1016/S1097-2765(00)00073-3.

Turner N., Grose R. 2010. Fibroblast growth factor signalling: from development to cancer. Nature Reviews Cancer 10:116-129. DOI: 10.1038/nrc2780.

Wagner S., Klepsch MM., Schlegel S., Appel A., Draheim R., Tarry M., Högbom M., Wijk KJ van., Slotboom DJ., Persson JO., Gier J-W de. 2008. Tuning Escherichia coli for membrane protein overexpression. Proceedings of the National Academy of Sciences 105:14371-14376. DOI: 10.1073/pnas.0804090105.

Wang F., Kan M., Yan G., Xu J., McKeehan WL. 1995. Alternately spliced NH2-terminal immunoglobulin-like Loop I in the ectodomain of the fibroblast growth factor (FGF) 
464

465

466

467

468

469

470

471

472

473

474

475

476

477

478

receptor 1 lowers affinity for both heparin and FGF-1. Journal of Biological Chemistry 270:10231-10235.

Yeh BK., Eliseenkova AV., Plotnikov AN., Green D., Pinnell J., Polat T., Gritli-Linde A., Linhardt RJ., Mohammadi M. 2002. Structural Basis for Activation of Fibroblast Growth Factor Signaling by Sucrose Octasulfate. Molecular and Cellular Biology 22:7184-7192. DOI: 10.1128/MCB.22.20.7184-7192.2002.

Yu K., Herr AB., Waksman G., Ornitz DM. 2000. Loss of fibroblast growth factor receptor 2 ligand-binding specificity in Apert syndrome. Proceedings of the National Academy of Sciences 97:14536-14541. DOI: 10.1073/pnas.97.26.14536.

Zhang F., Zhang Z., Lin X., Beenken A., Eliseenkova AV., Mohammadi M., Linhardt RJ. 2009. Compositional Analysis of Heparin/Heparan Sulfate Interacting with Fibroblast Growth Factor·Fibroblast Growth Factor Receptor Complexes. Biochemistry 48:8379-8386.

DOI: 10.1021/bi9006379. 